\title{
miR-21 plays a pivotal role in gastric cancer pathogenesis and progression
}

\author{
Zhiyu Zhang ${ }^{1, *}$, Zejuan Li ${ }^{2, *}$, Caiping Gao ${ }^{1}$, Ping Chen², Jianjun Chen² ${ }^{2}$ Wenzhong Liu ${ }^{1}$, Shudong Xiao ${ }^{1}$ and Hong Lu
}

Gastric cancer causes nearly one million deaths worldwide per year. Although Helicobacter pylori infection is the main risk factor, in about $80 \%$ or more of gastric cancers, the molecular pathway underlying $H$. pylori infection leading to the development of gastric cancers remains unclear. Recently accumulating evidence suggests that microRNAs (miRNAs) may regulate diverse biological processes and may be important in tumorigenesis. miR-21 has been frequently observed to be aberrantly overexpressed in various tumors. Using TaqMan quantitative real-time PCR, we confirmed that miR-21 was significantly overexpressed in human gastric cancer tissues and cell lines. Remarkably, miR-21 was also significantly overexpressed in $\mathrm{H}$. pylori-infected gastric mucosa, implying that overexpression of miR-21 in gastric cancer may be due in part to $H$. pylori infection. More importantly, we showed that forced expression of miR-21 significantly enhanced cell proliferation and invasion in AGS cells, a human gastric cancer cell line, whereas knockdown of miR-21 by inhibitor caused a significant reduction in cell proliferation and a significant increase in apoptosis. Furthermore, we demonstrated that knockdown of miR-21 significantly decreased cell invasion and migration of AGS cells. Finally, we showed that RECK, a known tumor suppressor in gastric cancer, is a bona fide target of miR-21. Taken together, miR-21 may be important in the initiation and progression of gastric cancers as an oncomiR, likely through regulating RECK. Our findings suggest a potential regulatory pathway in which $H$. pylori infection upregulates expression of miR-21, which in turn downregulates $R E C K$, and then leads to the development of gastric cancer.

Laboratory Investigation (2008) 88, 1358-1366; doi:10.1038/labinvest.2008.94; published online 15 September 2008

KEYWORDS: gastric cancer; H. pylori infection; miR-21; RECK

Gastric cancer, the second most common cancer in the world, causes nearly one million deaths worldwide per year. Although the etiology of gastric carcinogenesis is thought to be multifactorial, Helicobacter pylori ( $H$. pylori)-related gastric mucosal inflammation seems to be the most important trigger. ${ }^{1} H$. pylori is the main risk factor in about $80 \%$ or more of gastric cancers. The role of $H$. pylori on gastroduodenal diseases has been proposed, ${ }^{2}$ but the detailed molecular pathway remains unclear.

MicroRNAs (miRNAs, miRs) are a new class of small noncoding RNA that regulates the expression of target genes through translational repression or mRNA cleavage/decay. ${ }^{3,4}$ Genome-wide studies have demonstrated that miRNA genes are frequently located at cancer-associated genomic regions or in fragile sites, and in minimal regions of loss of heterozygosity or of amplifications, or in common breakpoint regions, indicating the potential roles of miRNAs in tumorigenesis. ${ }^{5}$ Aberrant miRNA expression has also been frequently reported in various tumors such as breast cancer, leukemia, lung cancer, and colon cancer, ${ }^{6-8}$ indicating that there is a close correlation between miRNAs and human malignancy. miR-21 has been frequently reported to be aberrantly overexpressed in diverse tumors. ${ }^{9-16}$ For example, Chan et $a l^{11}$ reported that expression of miR-21 was remarkably elevated in human glioblastoma and contributed to the malignant phenotype by blocking expression of critical apoptosis-related genes. Si et $a l^{14}$ found that miR-21 was highly expressed in breast tumors and found that the antimiR-21-mediated cell growth inhibition was associated with increased apoptosis and decreased cell proliferation. miR-21 was also reported to be overexpressed in malignant cholangiocyte; inhibition of miR-21 increased sensitivity to

\footnotetext{
'Department of Gastroenterology, Shanghai Renji Hospital, Shanghai Institute of Digestive Disease, Shanghai Jiaotong University School of Medicine, Shanghai, China and ${ }^{2}$ Department of Medicine, University of Chicago, Chicago, IL, USA

Correspondence: Dr H Lu, MD, PhD, Department of Gastroenterology, Shanghai Renji Hospital, Shanghai Institute of Digestive Disease, Shanghai Jiaotong University School of Medicine, 145 Shan-dong Zhong Road, Shanghai 200001, China.

E-mail: honglu02@yahoo.com

*These authors contributed equally to this work.

Received 23 March 2008; revised 6 July 2008; accepted 8 July 2008
} 
gemcitabine. ${ }^{16}$ Previously, in a large-scale miRNAs microarray assay, Volinia et al ${ }^{12}$ observed a significant overexpression of miR-21 in diverse solid tumors including gastric cancer. However, the role and relevant pathway of miR-21 in gastric carcinogenesis is largely unknown. In a real-time PCR analysis, we observed a significant overexpression of miR-21 in gastric cancer tissue and cell lines, as well as in $H$. pylori-infected tissue samples. The effect of miR-21 on apoptosis, proliferation, migration, and invasion ability were further investigated. Moreover, we identified RECK (reversion-inducing cysteine-rich protein with Kazal motifs), a tumor suppressor gene, as a target of miR-21. Our results suggested that $H$. pylori may function as an initiator in the process of gastric tumor genesis by regulating miR-21 expression, and thereby manipulating miR-21 target network(s).

\section{MATERIALS AND METHODS}

\section{Cell Lines, $H$. pylori Strain, and Growth Conditions}

Human gastric cancer cell line AGS was cultured in F12k medium with $10 \%$ fetal bovine serum (FBS). Other gastric cancer cell lines SGC7901, MKN45, MKN28, and a nonmalignant gastric epithelium cell line GES-1 were cultured in RPMI-1640 medium with 10\% FBS. HEK-293T cell was cultured in Dulbecco's modified Eagle's medium supplemented with $10 \%$ FBS. All cell lines were incubated at $37^{\circ} \mathrm{C}$ in $5 \% \mathrm{CO}_{2}$.

H. pylori strain NCTC 11637 was maintained on brain heart infusion agar medium (OXID, Basingstoke, UK) containing $5 \%$ sheep blood incubated at $37^{\circ} \mathrm{C}$ in $5 \% \mathrm{O}_{2}$ for a minimum of two and a maximum of four passages from frozen stocks. $H$. pylori bacteria were added to cultured AGS cells at ratio of 1:10 and cocultured till total RNA was extracted at $12,24,48$, and $72 \mathrm{~h}$ after $H$. pylori induction.

\section{Patients and Tissue Samples}

A total of 10 gastric cancer tissue samples were surgically obtained from patients in Shanghai Renji Hospital, China, and diagnosed by an independent pathologist. A total of 12 H. pylori-infected tissue samples and 8 normal gastric epithelium tissues were collected from the subjects who were referred for gastroscopy. The inflammation degree in all samples was verified by pathology. Informed consent was taken from all subjects and the Institute Ethics Committee approved the study protocol.

\section{TaqMan Quantitative Real-Time PCR Analysis of Mature miRNAs}

Total RNA isolated by TRIzol Reagent (Invitrogen, Carlsbad, CA, USA) was treated with the Turbo DNA free kit (Ambion, Austin, TX, USA) to eliminate the genome DNA contamination. TaqMan stem-loop real-time PCR method was used to assess the expression of miR-21 with kits from Applied Biosystems (Foster City, CA, USA). In each sample, we calculated a $\Delta C_{\mathrm{t}}$ (target-reference), which is equal to the difference between threshold cycles for miR-21 (ie, target) and the threshold cycle for U6 RNA (ie, reference) (ie, $\Delta C_{t}$ (target-reference $)=C_{\mathrm{t}}$ target $-C_{\mathrm{t}}$ reference). The fold-change between patient or cell sample and a normal control for miR-21 was calculated with the $2^{-\Delta \Delta C_{\mathrm{t}}}$ method, in which $\Delta \Delta C_{\mathrm{t}}=\Delta C_{\mathrm{t}}$ (target-reference) (in patient sample or cell line) $-\Delta C_{\mathrm{t}}$ (target-reference) (in normal control). Real-time PCR was repeated in triplicate for each sample, an average $2^{-\Delta \Delta C_{t}}$ value along with its s.d. was calculated for each sample relative to the normal control for expression of miR-21.

\section{Real-Time PCR for RECK}

Total RNA $(1 \mu \mathrm{g})$ was reverse transcribed using reverse transcription kit (Promega, Madison, WI, USA). Quantitative real-time PCR was performed on ABI 7700 PCR Instrument (ABI, Foster City, CA, USA) with a SYBR Green-real-time PCR master mix kit (Toyobo, Tokyo, Japan) for detection of RECK. GAPDH was used as endogenous control (ie, reference gene). Forward $(\mathrm{F})$ and reverse $(\mathrm{R})$ primer sequences were as follows: RECK (F) $5^{\prime}$-agcaaccgagcccgtatgt- $3^{\prime}, R E C K$ (R) $5^{\prime}$-ccgagtaggcagcacacaca- $3^{\prime}$, GAPDH $(\mathrm{F}) \quad 5^{\prime}$-acggatttggtcgtat tgggc- $3^{\prime}$, and GAPDH (R) $5^{\prime}$-ttgacggtgccatggaatttg- $3^{\prime}$. PCR was performed for $15 \mathrm{~s}$ at $95^{\circ} \mathrm{C}$ and $1 \mathrm{~min}$ at $60^{\circ} \mathrm{C}$ for 40 cycles.

\section{Cell Transfection}

To create the miR-21 expression vector, the precursor sequence of miR-21 (172bp) was amplified by PCR using primers as follows: miR-21-precursor-F $\left(5^{\prime}\right.$-tacctcgagtgt ctgcttgttttgcct- $\left.3^{\prime}\right)$ and miR-21-precursor-R ( $5^{\prime}$-tacgaattctgttta aatgagaacatt- $3^{\prime}$ ) from human normal bone marrow mononuclear cells and then cloned into the MSCVpuro vector (Clontech). AGS cells were grown on six-well plate to confluence. Each vector ( $3 \mu \mathrm{g}$ per well) was transfected using Fugene HD transfection reagent (Roche Diagnostics). MSCVpuro vector was used as a negative control. AntimiR-21 inhibitor ( $5^{\prime}$-ucaacaucagucugauaagcua- $\left.3^{\prime}\right)$ and mismatched sequence negative control oligonucleotide $\left(5^{\prime}\right.$-cagu acuuuuguguaguacaa- $3^{\prime}$ ) were synthesized by GenePharma, Shanghai, transfected into AGS cells $(200 \mathrm{nmol}$ per well) using Oligofectamine reagent (Invitrogen). Transfection efficiency was evaluated by GFP expression in control vector or real-time PCR (Supplementary Materials).

\section{Cell Proliferation Assay}

A total of $10^{4}$ AGS cells per well were plated in 96-well plates before transfection and cultured for $24 \mathrm{~h}$ in normal conditions. They were then transfected with mscvpuro-miR-21 or antimiR-21 inhibitor along with paired negative controls. Cell proliferation was assessed $48 \mathrm{~h}$ later using Cell Counting Kit 8 (Dojindo, Tokyo, Japan) according to manufacturer's protocol.

\section{Apoptosis Assay}

At $72 \mathrm{~h}$ after transfection, apoptosis was detected using Annexin V-FITC Apoptosis Detection Kit (Biovision, USA). Results were calculated by the percentage of apoptotic cells in all cells counted. 
a

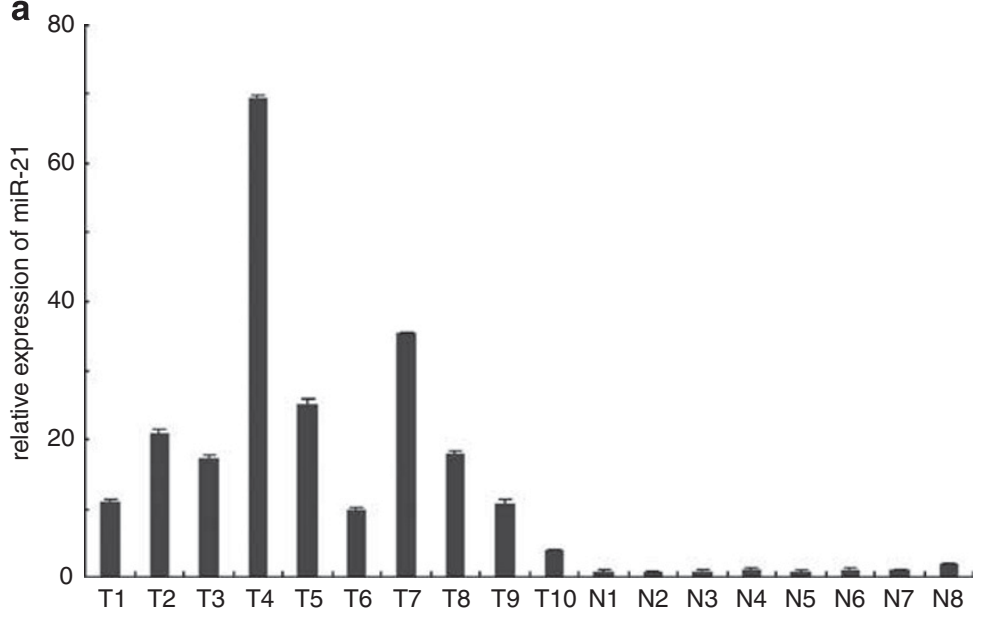

b

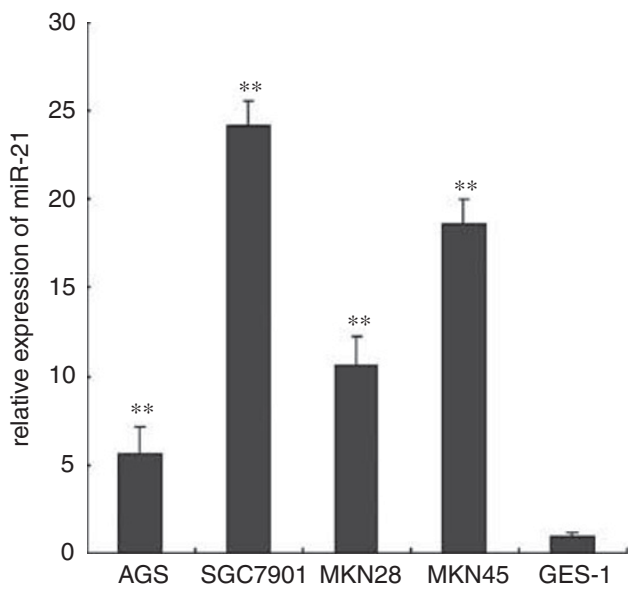

Figure 1 Overexpression of miR-21 in gastric cancer tissues and cell lines compared to the corresponding normal controls. (a) Comparison of expression level of miR-21 between gastric cancer tissue and normal tissue samples; (b) comparison of expression level of miR-21 between gastric cancer cell lines AGS, SGC7901, MKN28, MKN-45, and a control cell line (GES-1). Relative expression level of miR-21 was determined by TaqMan real-time PCR, and all data were normalized by U6 RNA. Means \pm s.d. are shown. ${ }^{* *} P<0.01$.
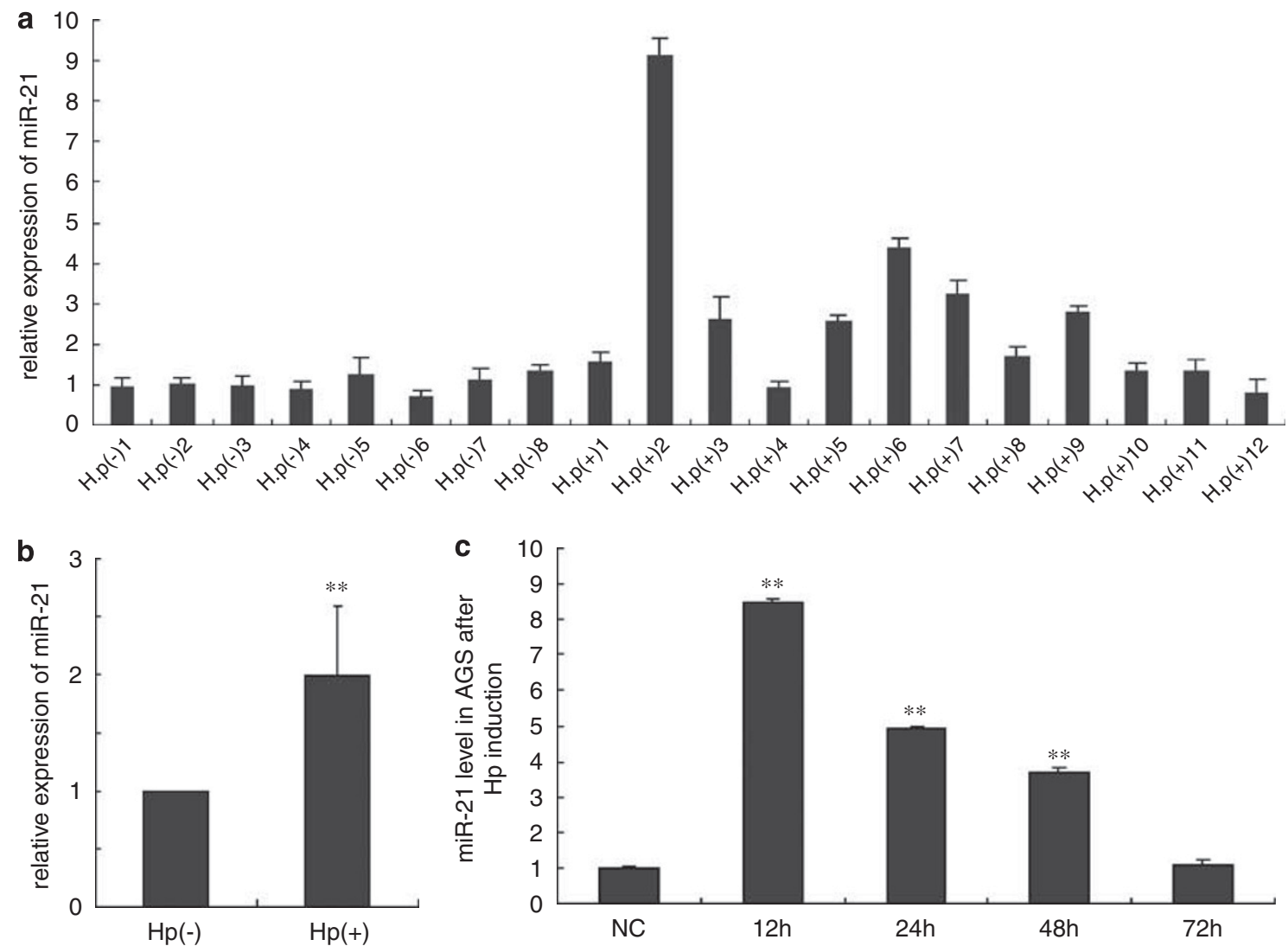

Figure 2 Overexpression of miR-21 in samples infected with chronic H. pylori or in AGS cells infected by H. pylori. (a) Expression level of miR-21 in individual gastric epithelium samples. (b) Comparison of the average expression level of miR-21 between $H$. pylori-infected samples and normal control samples. ${ }^{* *} P<0.05$. (c) Relative miR-21 expression in AGS cells after $H$. pylori introduction. ${ }^{* * P}<0.05$.

\section{Luciferase Reporter Assay}

A fragment of the $3^{\prime}$-UTR of RECK (primer F: $5^{\prime}$-tcgac tagtgttctgtctgactttc- $3^{\prime}$, primer R: $5^{\prime}$-tgaaagctttaagtgcccatttttc- $3^{\prime}$ ), which contains the putative miR-21-binding sequence, was cloned into a luciferase reporter construct (pMIR-Report plasmid; Ambion). The wild-type or a mutated $3^{\prime} \mathrm{UTR}$ of RECK construct (GeneTailor ${ }^{\mathrm{TM}}$ Site-Directed Mutagenesis System; Invitrogen; primer F: 5'-gtttcacagtttgaacgggatgctttga 

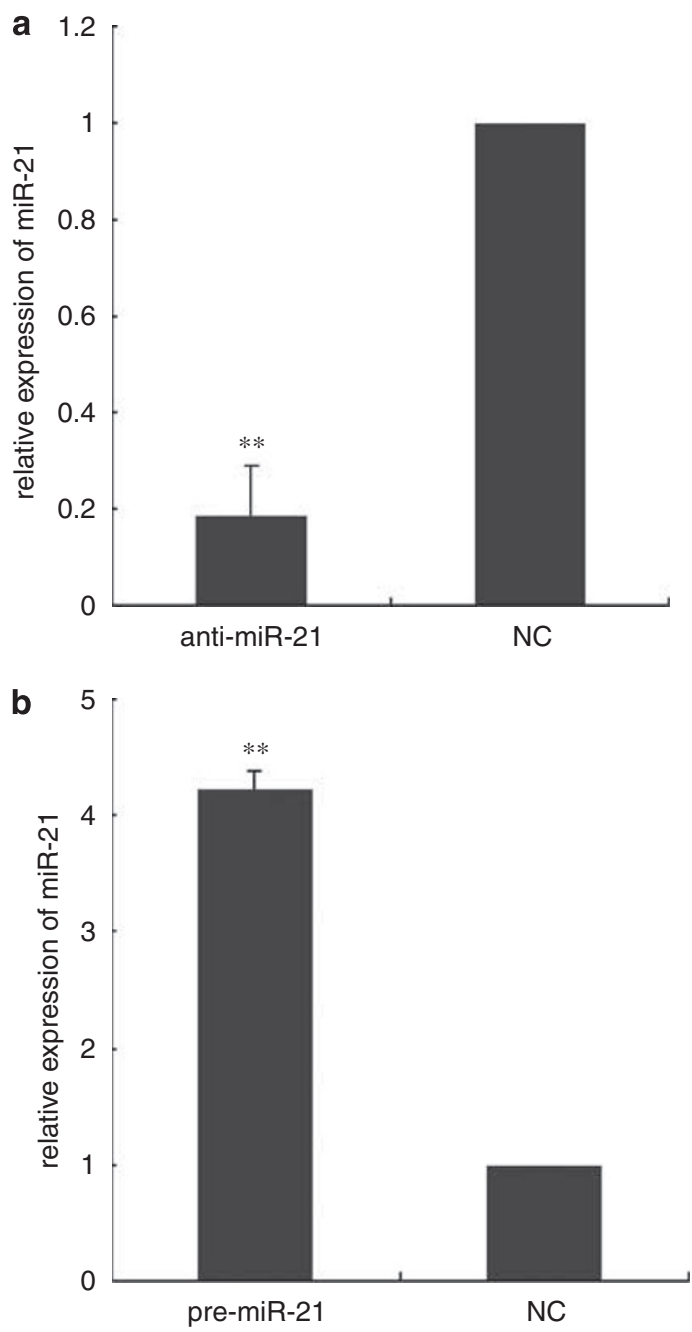

Figure 3 miR-21 regulates cell proliferation. (a) Forced expression of miR21 significantly enhanced cell proliferation in AGS cell. (b) Knockdown of miR-21 significantly decreased cell proliferation in AGS cell. Data calculated from two separate experiments of five replicates each. Means \pm s.d. are shown. ${ }^{* *} p<0.05$.

tgtaatacttc- $3^{\prime}$, primer R: $5^{\prime}$-ttcaaactgtgaaacacattcaaagtattcaat- $3^{\prime}$ ) and MSCVpuro-miR-21 were cotransfected into HEK-293T cells. Luciferase was measured $42 \mathrm{~h}$ after transfection. The firefly luciferase activity was then normalized to $\beta$-galactosidase activity. Noncluster-related miR-152 expression plasmid (MSCVpuro-miR-152) and MSCVpuro were cotransfected with the RECK $3^{\prime}$-UTR construct, respectively, into HEK-293T cells as controls. Experiments were repeated three times independently.

\section{Western Blotting}

Western blot was performed according to standard procedures. Total protein was isolated from tumor samples and corresponding normal tissues or AGS cells. The concentration was measured by BCA protein assay kit (Pierce, USA). The membrane was first incubated with antibody against RECK (R\&D Systems Inc.), then with anti- $\beta$-actin antibody
(Sigma) as a loading control. Signals were detected by secondary antibodies labeled with HRP and signal intensity was determined by Image J software.

\section{Matrigel Invasion Assay}

Matrigel invasive assays were performed using Thincert Transwell chamber (Greiner Bio-One, Germany) (pore size $8 \mathrm{~mm})$ coated with Matrigel (50 $\mu$ l per filter) (BD, USA) as described in the manufacturer's protocol. The lower chamber was filled with $0.6 \mathrm{ml}$ of F12k medium containing $10 \%$ FBS. AGS cells were serum-starved overnight, and then resuspended in F12k medium without FBS. Cell suspension $(100 \mu \mathrm{l})$ containing $1 \times 10^{5}$ cells was added to the upper chamber. After $24 \mathrm{~h}$ incubation, noninvaded cells on the upper surface of the membrane were scraped off by cotton tip. The migrant cells attached to the lower surface were fixed in $90 \%$ alcohol and followed by crystal violet stain. The number of migrated cells on the lower surface of the membrane was counted under a microscope in 10 fields with magnification of $\times 400$.

\section{Scratch Wound-Healing Motility Assays}

When AGS cells were seeded and grown to confluence, a scratch was set with a pipette tip running though the dish and cultured under standard conditions for $24 \mathrm{~h}$. Plates were washed twice with fresh medium to remove nonadherent cells and then photographed. The cell migration was evaluated by counting cells that migrated from the wound edge.

\section{Statistical Analysis}

Data are expressed as the mean \pm s.d. unless otherwise noted. The differences between groups were analyzed using a twotailed Student's $t$-test when only two groups were present and the null hypothesis was rejected at the 0.05 level unless otherwise specified.

\section{RESULTS}

miR-21 is Aberrantly Overexpressed in Gastric Cancer Tissue Samples and Cell Lines

Through TaqMan quantitative real-time PCR analysis, we observed that miR-21 expressed at a significantly higher level $(P<0.01 ; 18.25$-fold on average $)$ in gastric cancer tissue samples than in normal tissue samples (Figure 1a). A significant overexpression of miR-21 is also observed in all four gastric cancer cell lines relative to a nonmalignant gastric cell line GES-1 (Figure 1b; $P<0.01 ; 14.8$-fold change on average).

\section{H. pylori Infection May Cause the Upregulation of miR-21 in Gastric Epithelium Tissue Samples and AGS Cells}

Because H. pylori infection is considered to be the factor most responsible for gastric cancers, we hypothesized that the upregulation of miR-21 in gastric mucosa might be associated with $H$. pylori infection. We then investigated the expression of miR-21 in 12 chronic $H$. pylori-infected gastric tissue samples and 8 normal tissue samples using real-time 
a

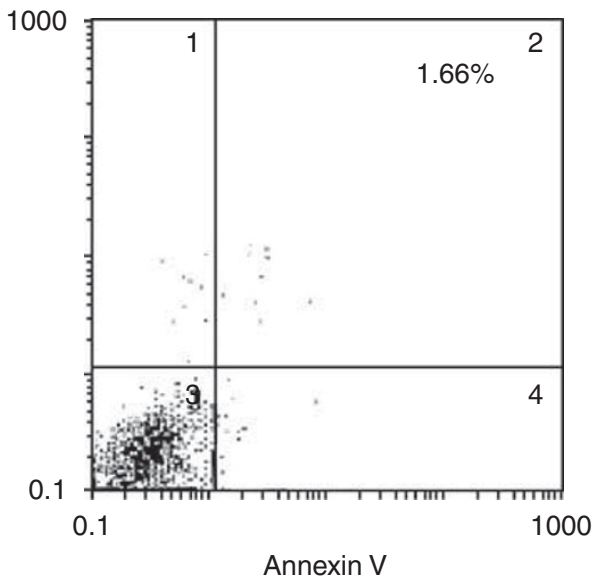

b

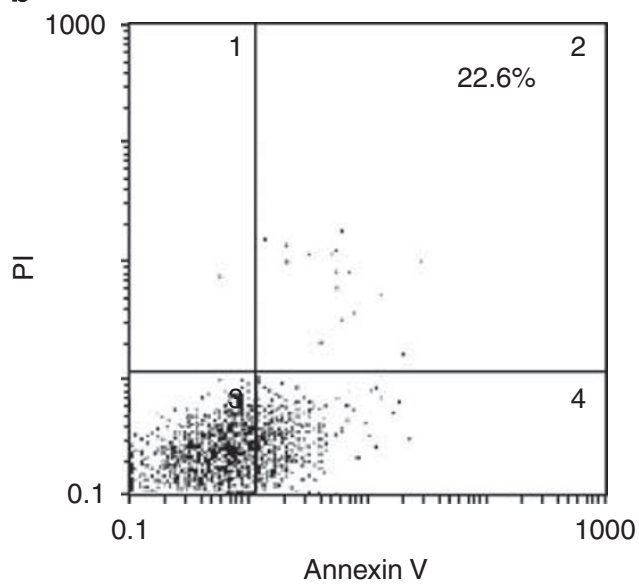

Figure 4 Knockdown of miR-21 increases apoptosis in AGS cells. The proportion of apoptosis cells induced by transfection of anti-miR-21 inhibitor (b) is significantly greater $(22.6$ vs $1.66 \% ; P<0.01)$ than that induced by transfection of mismatched sequence miRNA negative control (a).

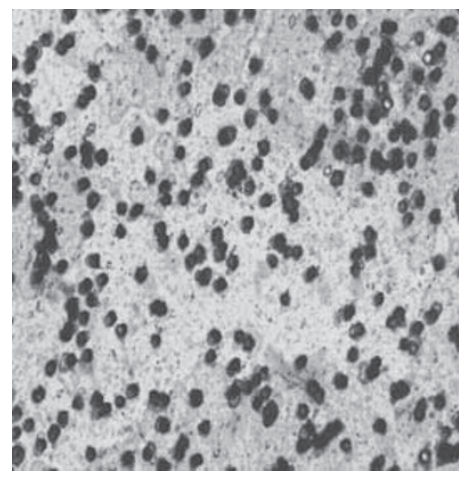

d

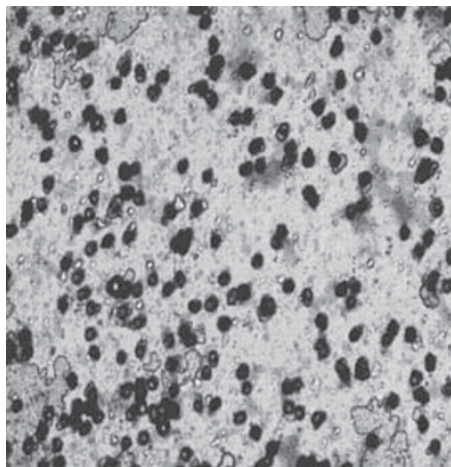

b

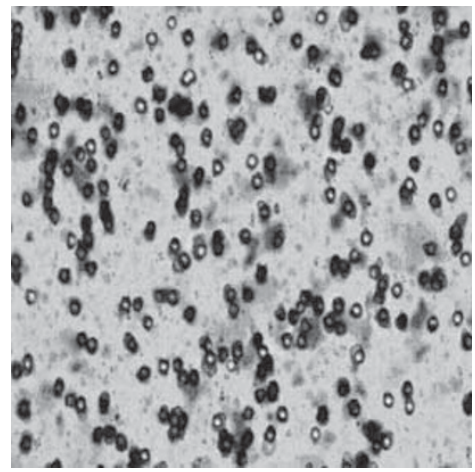

e

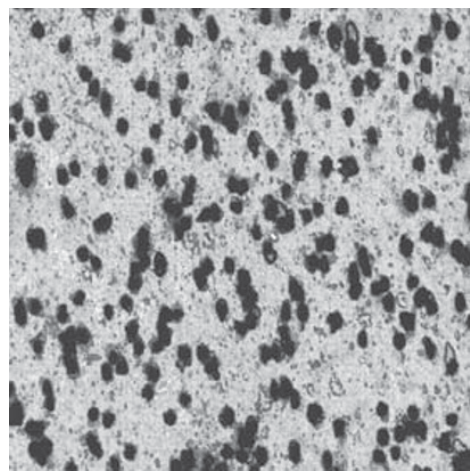

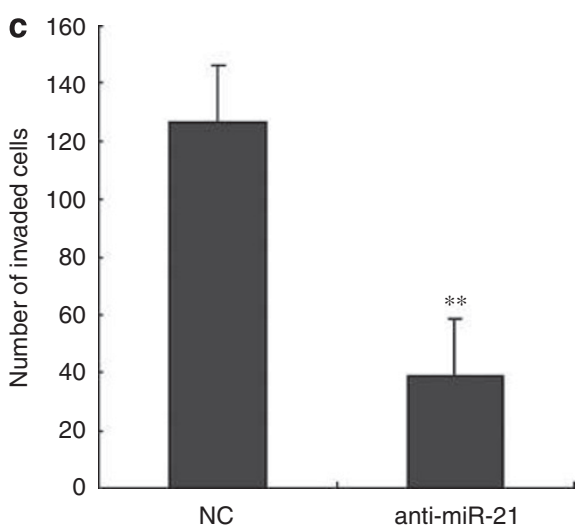

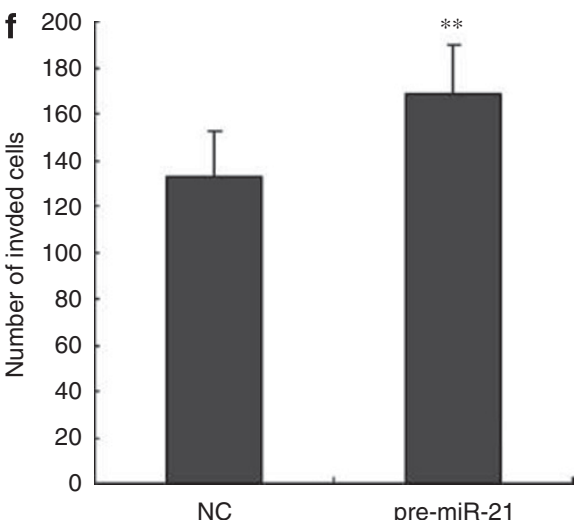

Figure 5 miR-21 regulates cell invasion ability in AGS cells. (a) AGS cells that invaded through Matrigel-coated membrane after transfection with negative control miRNA and (b) transfection with anti-miR-21 inhibitor. (c) Quantification of AGS cells that invaded through Matrigel-coated membrane after transfection with anti-miR-21 inhibitor or negative control. (d) AGS cells that invaded through Matrigel-coated membrane after transfection with negative control vector and (e) transfeection with MSCVpuro-miR-21 (pre-miR-21). (f) Quantification of AGS cells that invaded through Matrigel-coated membrane after transfection with MSCVpuro-miR-21 or negative control. ${ }^{* *} P<0.05$.

PCR method. As expected, miR-21 was overexpressed $(P<0.05$; twofold on average $)$ in $H$. pylori-infected tissue samples compared with normal tissue samples (Figure 2a and b). To confirm the results above, we reexamined miR-21 expression in AGS cells induced by $H$. pylori. The expression of miR-21 increased to the highest level at $12 \mathrm{~h}$ of $\mathrm{H}$. pylori induction. Although the expression of miR-21 was also high at 24 and $48 \mathrm{~h}$, then returned to normal level at $72 \mathrm{~h}$ after 
a

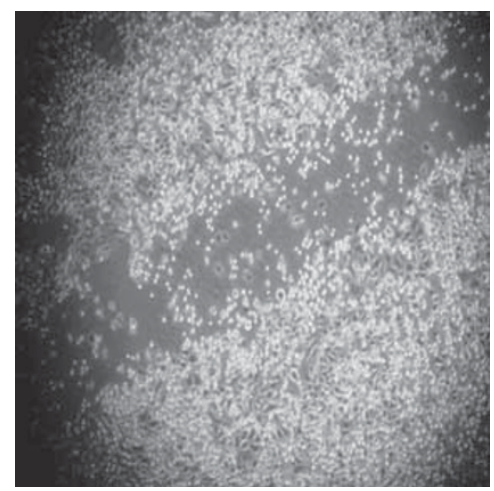

d

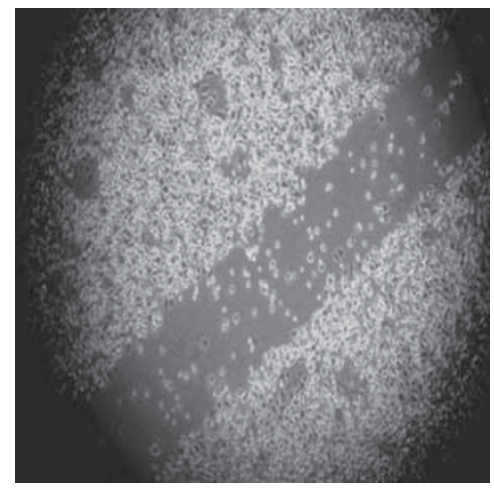

b

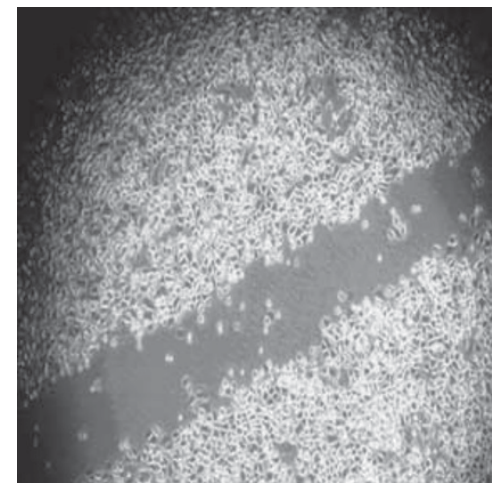

e

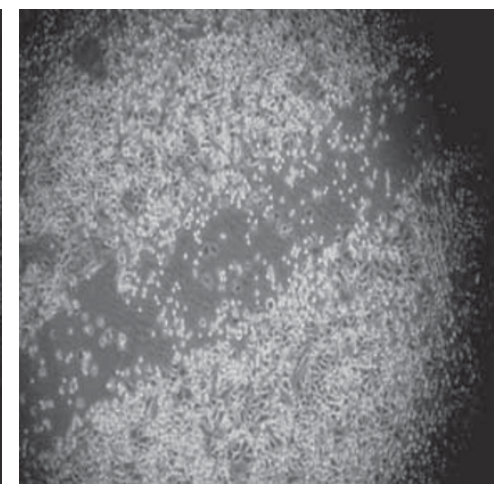

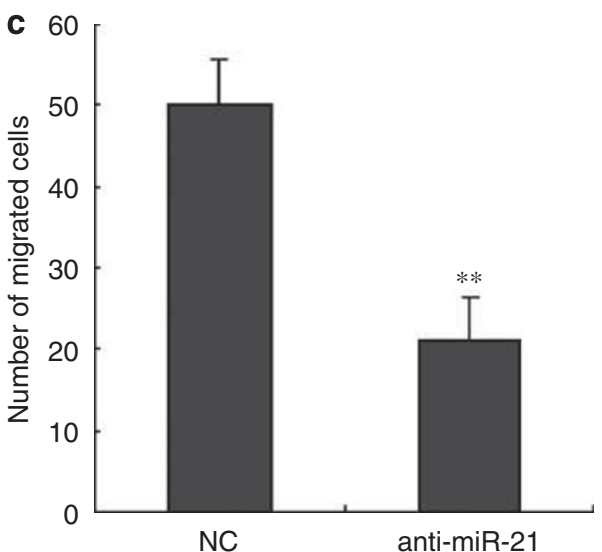

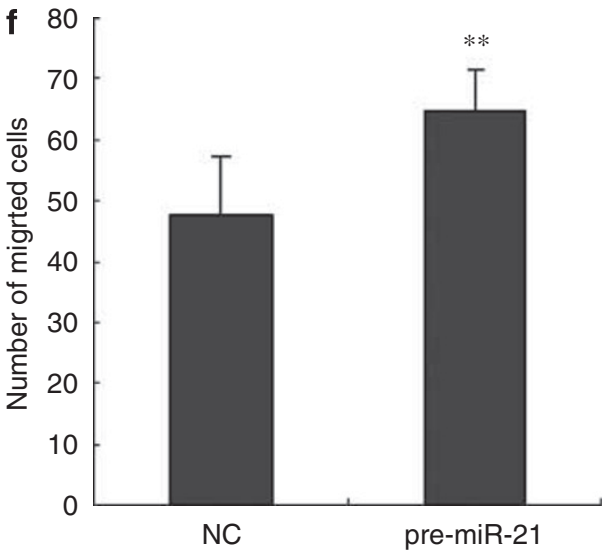

Figure 6 miR-21 influences AGS cell migration ability. (a) Migration of AGS cells transfected with negative control miRNA and (b) transfection with anti-miR-21 inhibitor. (c) Quantification of AGS cells that migrated from the edge of scratch transfected with anti-miR-21 inhibitor and negative control. (d) Migration of AGS cells transfected with negative control vector and (e) transfection with MSCVpuro-miR-21 (pre-miR-21). (f) Quantification of AGS cells that migrated from the edge of scratch after transfection with MSCVpuro-miR-21 or negative control. ${ }^{* * P<0.05}$.

H. pylori treatment (Figure 2c; $P<0.05$ ). This suggested that overexpression of miR-21 in gastric cancer cells might be, at least partly, caused by $H$. pylori infection.

\section{miR-21 Increases Cell Proliferation and Inhibits Cell Apoptosis}

We further investigated the potential oncogenic activity of miR-21. Aberrant cell proliferation is a hallmark of cancers. First, we tested miR-21 expression in AGS cell line using TaqMan real-time PCR. It showed an increase or decrease after transfected with MSCVpuro-miR-21 or anti-miR-21 inhibitor (Supplementary Materials). We observed a significant increase $(P<0.05)$ in proliferation after transfection of MSCVpuro-miR-21 (Figure 3a). In contrast, anti-miR-21 inhibitor significantly $(P<0.05)$ decreased cell proliferation (Figure $3 \mathrm{~b}$ ). These data indicate that cell proliferation can be significantly enhanced by increase of miR-21 expression.

We further investigate the effect of miR-21 on apoptosis and found that apoptosis was increased dramatically (Figure 4; $>13.6$-fold; $P<0.01$ ) in AGS cells $72 \mathrm{~h}$ after transfection with
anti-miR-21 inhibitor, suggesting that miR-21 may function as a strong antiapoptotic factor in human gastric cancer cells.

\section{miR-21 Regulate Gastric Cancer Cell Invasion and Migration In Vitro}

In an in vitro cell invasion assay (see 'Materials and Methods' for details), we observed that cell invasion was significantly suppressed $(P<0.05$; $\sim 3$-fold) by transfection of anti-miR21 inhibitor (anti-miR-21 group, $39 \pm 11$ cells per HP; negative control group, $133 \pm 21$ cells per HP) (Figure $5 \mathrm{a}-\mathrm{c}$ ) or remarkably strengthened $(P<0.05)$ by transfection of MSCVpuro-miR-21 (pre-miR-21 group, $169 \pm 20$ cells per HP; negative control group, $127 \pm 25$ cells per HP) (Figure $5 \mathrm{~d}-\mathrm{f})$. In cell scratch wound healing test, the number of AGS cells migrated to the scratched area was significantly fewer $(P<0.05 ;<2$-fold $)$ in cells transfected with anti-miR-21 inhibitor $(21 \pm 6$ cells per HP) than in those transfected with negative control ( $50 \pm 6$ cells per HP) (Figure $6 \mathrm{a}-\mathrm{c}$ ). However, if overexpressed miR-21, the number of the migrated cells $(65 \pm 10$ cells per $\mathrm{HP}$ ) were significantly more than negative control (48 \pm 7 cells per HP) (Figure 6d-f) 

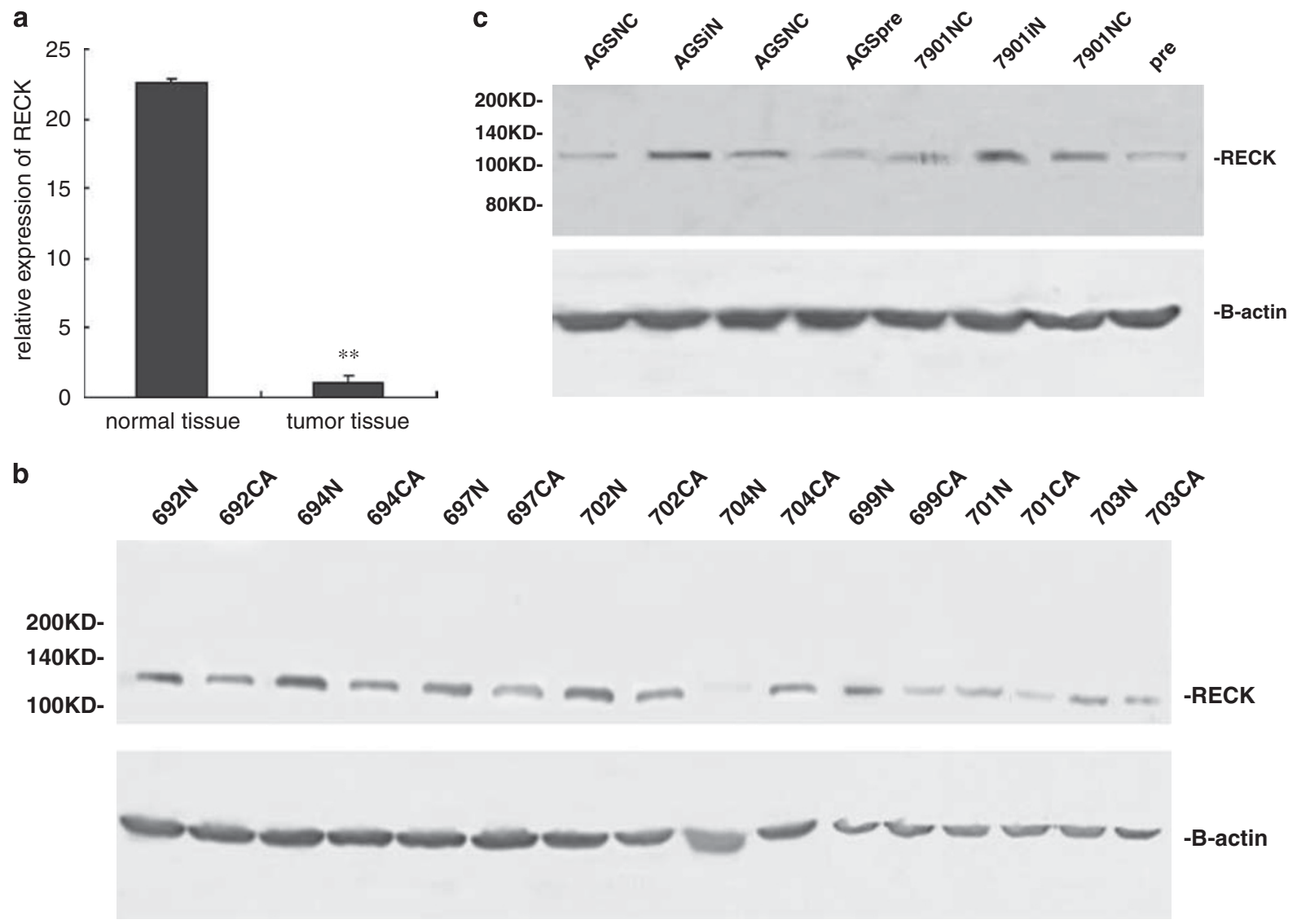

Figure 7 RECK is a target of miR-21. (a) Relative expression level of RECK in gastric cancer and normal tissues normalized by GAPDH detected by real-time PCR. (b) Expression level of RECK in tumor and normal tissues detected by western blotting. (c) RECK expression level in AGS or SGC7901 cell after transfection with anti-miR-21 inhibitor or MSCVpuro-miR-21 (pre-miR-21) detected by western blotting. (d) Predicted consequential pairing of target $3^{\prime}$-UTR region of RECK (wild type or mutated) and miR-21 mature sequence. (e) Luciferase activity on the presence of both wild-type RECK $3^{\prime} U T R$ or mutant and miR-21 (ie, $3^{\prime} U T R+$ miR-21) were compared with those of the controls (ie, $3^{\prime} U T R+$ vacant vector and $3^{\prime} U T R+$ miR-152). ${ }^{* *} P<0.05$.

$(P<0.05)$. These results indicate that miR-21 may be important in the progression of gastric cancer through enhancing cell invasion and migration.

\section{RECK is a Target of miR-21}

We then searched for the potential targets of miR-21 using all four currently available major prediction programs, including TargetScan, ${ }^{17}$ Miranda, ${ }^{18}$ PicTar, ${ }^{19}$ and MAMI (http:// mami.med.harvard.edu/). We found that RECK, a tumor suppressor gene, was predicted by all the four programs as a target of miR-21. As shown in Figure 7, expression of RECK is significantly lower in gastric tumor samples than in the normal controls at both transcriptional level (Figure 7a; $P<0.05$ ) and protein level (Figure $7 \mathrm{~b} ; P<0.05$; average $<2.0$-fold). In AGS or SGC7901 cells, RECK can be regulated negatively by miR-21 at protein level (Figure $7 \mathrm{c} ; P<0.05$ ). Furthermore, we performed a luciferase reporter assay and observed a significant decrease $(P<0.05)$ in luciferase activity in the presence of MSCVpuro-miR-21 in 293T cell compared with the controls. In addition, to validate whether RECK is a direct target of miR-21, we mutated the miR-21 binding site in the $3^{\prime} \mathrm{UTR}$ of RECK (in the reporter plasmid) and observed loss of repression (Figure $7 \mathrm{~d}$ and e). These results all indicate $R E C K$ is a direct target of miR-21.

\section{DISCUSSION}

Although miRNAs have been extensively studied in different types of cancers in recent years, the knowledge of the aberrant expression and potential role of miRNAs in gastric cancer is largely lacking. Here we showed that expression of miR-21 was significantly increased in gastric tumor tissues and cancer cell lines compared with normal controls, which is consistent with the finding of Volinia et al. ${ }^{12}$ Our data also indicate that the upregulation of miR-21 in gastric cancer is probably related to $H$. pylori infection. Moreover, we also found that $H$. pylori infection could induce miR-21 elevation in vitro.

Consistent with previous findings from other cancers, ${ }^{11,14,16}$ in gastric cancer, we also found that miR-21 could also remarkably increase cell proliferation and inhibit apoptosis. In addition, we showed that knockdown of miR-21 could dramatically decrease cell invasion and migration in gastric cancer cells; on the contrary, overexpression of miR-21 
d

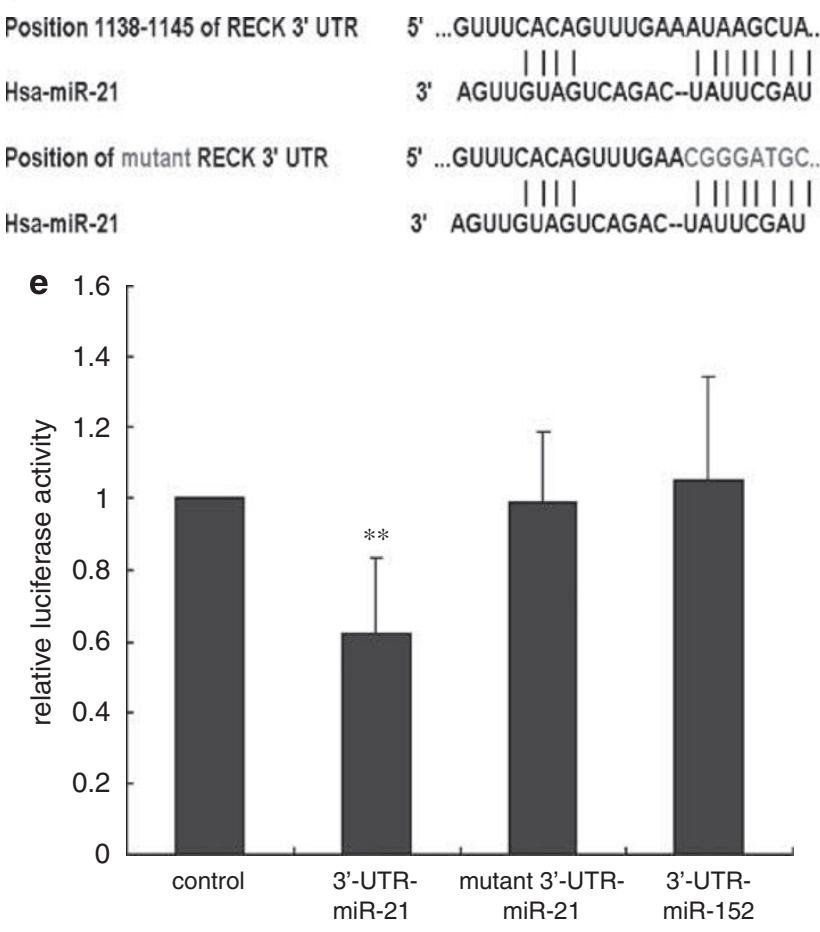

Figure 7 continued.

could lead the opposite effect in AGS cells. Thus, our data suggest that miR-21 may be important in the development of gastric cancer. In an in vivo study of pancreatic tumors, a relationship between liver metastasis and miR-21 has also been reported. ${ }^{20}$ Therefore, our results together with those of others ${ }^{11,14,16,20}$ indicate that miR-21 has multiple functions in diverse cancers and could be recognized as an important oncomiR. $^{21}$

In addition, we identified a tumor suppressor gene, RECK, as a target of miR-21. RECK has been reported as a tumor suppressor gene in gastric cancer and cell lines. ${ }^{22}$ It performs an important function in tumor metastasis and angiogenesis through modulating matrix metalloproteases (MMPs), including MMP9, MMP2, and MMP14, which are known to be involved in cancer progression. ${ }^{23}$ Our data suggested that RECK expression has a negative correlation with the expression of miR-21 in gastric tissues and cell lines at transcriptional level and protein level. Applying specific inhibitor of miR-21 can rescue RECK expression, negatively regulated gastric cancer cell metastasis. This is consistent with the previous result ${ }^{22}$ and promotes that the miR-21-RECK regulatory network is important in cancer pathogenesis and progression. Interestingly, Meng et al reported recently that miR-21 may target PTEN directly in human hepatocellular, and modulation of miR-21 altered focal adhesion kinase phosphorylation and expression of MMP2 and MMP9, both downstream mediators of PTEN involved in cell migration and invasion. ${ }^{10}$ Taken together, miR-21 may negatively regulate RECK and PTEN, which in turn alter focal adhesion kinase phosphorylation and expression of several MMPs, and thereby contribute to cancer cell migration and invasion. Besides RECK and PTEN, some other genes have also been identified as targets of miR-21. For example, Si et $a l^{14}$ reported that miR-21 modulated tumor genesis probably through regulation of genes such as BCL2. Zhu et al ${ }^{15}$ identified a tumor suppressor TPM-1 as a potential target of miR-21. Thus, the miR-21-mediated network might be much more complex than previously appreciated, and therefore, identification of all important targets and understanding of the relevant molecular pathways in various physiological and pathologic conditions will be very important for us to completely understand the biological functions of this miRNA.

H. pylori infection was thought to be the most important factor for gastric carcinogenesis from gastritis to atrophy, intestinal metaplasia, dysplasia, then to gastric cancer. But the pathogenic mechanism is still unknown. Here we have shown that expression of miR-21 was upregulated in the patients who got $H$. pylori infection, implying that $H$. pylori infection induces carcinogenesis in the stomach probably through altering expression of some oncomiRs such as miR-21. In conclusion, miR-21 is overexpressed in gastric cancer, and aberrant expression of miR-21 can alter multiple biological processes of human gastric cancer cells such as proliferation, apoptosis, migration, and invasion, probably through regulating RECK and other critical target genes. miR-21 can serve as a biomarker for gastric cancer, and an inhibitory strategy against miR-21 or miR-21/RECK interaction will have a strong rationale for treatment for gastric cancer. Moreover, eradiation of $H$. pylori may be a necessary tool for prevention of gastric cancer.

Supplementary Information accompanies the paper on the Laboratory Investigation website (http://www.laboratoryinvestigation.org)

\section{ACKNOWLEDGEMENTS}

This work was supported by the National Natural Science Foundation of China (30670940). We thank Dr Janet D Rowley and Mrs Mary Beth Neilly at the University of Chicago for their constructive suggestions and invaluable effort in manuscript editing and revision.

1. IARC. Schistosomes, liver flukes and Helicobacter pylori. IARC working group on the evaluation of carcinogenic risks to humans. IARC Monogr Eval Carcinog Risks Hum 1994;61:1-241.

2. Fidler IJ. The pathogenesis of cancer metastasis: the 'seed and soil' hypothesis revisited. Nat Rev Cancer 2003;3:453-458.

3. Peek Jr RM, Blaser MJ. Helicobacter pylori and gastrointestinal tract adenocarcinomas. Nat Rev Cancer 2002;2:28-37.

4. Pillai RS. MicroRNA function: multiple mechanisms for a tiny RNA? RNA 2005;11:1753-1761.

5. Zamore PD, Haley B. Ribo-gnome: the big world of small RNAs. Science 2005;309:1519-1524.

6. Calin GA, Sevignani C, Dumitru CD, et al. Human microRNA genes are frequently located at fragile sites and genomic regions involved in cancers. Proc Natl Acad Sci USA 2004;101:2999-3004.

7. Calin GA, Liu CG, Sevignani C, et al. MicroRNA profiling reveals distinct signatures in B cell chronic lymphocytic leukemias. Proc Natl Acad Sci USA 2004;101:11755-11760. 
8. Calin GA, Dumitru CD, Shimizu $M$, et al. Frequent deletions and down-regulation of micro- RNA genes miR15 and miR16 at $13 q 14$ in chronic lymphocytic leukemia. Proc Natl Acad Sci USA 2002;99:15524-15529.

9. Michael MZ, O' Connor SM, van Holst Pellekaan NG, et al. Reduced accumulation of specific microRNAs in colorectal neoplasia. Mol Cancer Res 2003;1:882-891.

10. Meng $\mathrm{F}$, Henson $\mathrm{R}$, Wehbe-Janek $\mathrm{H}$, et al. MicroRNA-21 regulates expression of the PTEN tumor suppressor gene in human hepatocellular cancer. Gastroenterology 2007;133:647-658.

11. Chan JA, Krichevsky AM, Kosik KS. MicroRNA-21 is an antiapoptotic factor in human glioblastoma cells. Cancer Res 2005;65: 6029-6033.

12. Volinia S, Calin GA, Liu CG, et al. A microRNA expression signature of human solid tumors defines cancer gene targets. Proc Natl Acad Sci USA 2006;103:2257-2261.

13. Lu J, Getz G, Miska EA, et al. MicroRNA expression profiles classify human cancers. Nature 2005;435:834-838.

14. Si ML, Zhu S, Wu H, et al. miR-21-mediated tumor growth. Oncogene 2007:26:2799-2803

15. Zhu S, Si ML, Wu H, et al. MicroRNA-21 targets the tumor suppressor gene tropomyosin 1 (TPM1). J Biol Chem 2007;282:14328-14336.
16. Meng $\mathrm{F}$, Henson $\mathrm{R}$, Lang $\mathrm{M}$, et al. Involvement of human micro-RNA in growth and response to chemotherapy in human cholangiocarcinoma cell lines. Gastroenterology 2006;130:2113-2129.

17. Lewis BP, Burge CB, Bartel DP. Conserved seed pairing, often flanked by adenosines, indicates that thousands of human genes are microRNA targets. Cell 2005;120:15-20.

18. Krek A, Grun D, Poy MN, et al. Combinatorial microRNA target predictions. Nat Genet 2005;37:495-500.

19. John B, Enright AJ, Aravin A, et al. Human MicroRNA targets. PLoS Biol 2004;2:e363.

20. Roldo C, Missiaglia E, Hagan JP, et al. MicroRNA expression abnormalities in pancreatic endocrine and acinar tumors are associated with distinctive pathologic features and clinical behavior. J Clin Oncol 2006;24:4677-4684.

21. Esquela-Kerscher A, Slack FJ. Oncomirs-microRNAs with a role in cancer. Nat Rev Cancer 2006;6:259-269.

22. Song SY, Son HJ, Nam E, et al. Expression of reversion-inducingcysteine-rich protein with Kazal motifs (RECK) as a prognostic indicator in gastric cancer. Eur J Cancer 2006;42:101-108.

23. Oh J, Takahashi R, Kondo S, et al. The membrane-anchored MMP inhibitor RECK is a key regulator of extracellular matrix integrity and angiogenesis. Cell 2001;107:789-800. 